\title{
Transcriptomic and rRNA:rDNA Signatures of Environmental versus Enteric Enterococcus faecalis Isolates under Oligotrophic Freshwater Conditions
}

\author{
(D) Brittany Suttner, ${ }^{a}$ (D) Minjae Kim, ${ }^{a *}$ Eric R. Johnston, ${ }^{a \S}$ Luis H. Orellana, ${ }^{\mathrm{a} \diamond}$ Carlos A. Ruiz-Perez, ${ }^{\mathrm{b}}$ (D) Luis M. Rodriguez-R, ${ }^{\mathrm{b} \infty}$ \\ Janet K. Hatt, ${ }^{a}$ Joe Brown, ${ }^{\text {a }}$ Jorge W. Santo Domingo, ${ }^{c}$ (D) Konstantinos T. Konstantinidis ${ }^{\mathrm{a}, \mathrm{b}}$ \\ aSchool of Civil and Environmental Engineering, Georgia Institute of Technology, Atlanta, Georgia, USA \\ bSchool of Biological Sciences, Georgia Institute of Technology, Atlanta, Georgia, USA \\ cU.S. Environmental Protection Agency, Center for Environmental Solution and Emergency Response, Cincinnati, Ohio, USA
}

ABSTRACT The use of enterococci as a fecal indicator bacterial group for public health risk assessment has been brought into question by recent studies showing that "naturalized" populations of Enterococcus faecalis exist in the extraenteric environment. The extent to which these naturalized $E$. faecalis organisms can confound water quality monitoring is unclear. To determine if strains isolated from different habitats display different survival strategies and responses, we compared the decay patterns of three $E$. faecalis isolates from the natural environment (environmental strains) against three human gut isolates (enteric strains) in laboratory mesocosms that simulate an oligotrophic, aerobic freshwater environment. Our results showed similar overall decay rates between enteric and environmental isolates based on viable plate and quantitative PCR ( $q P C R$ ) counts. However, the enteric isolates exhibited a spike in copy number ratios of $16 \mathrm{~S}$ rRNA gene transcripts to $16 \mathrm{~S}$ rRNA gene DNA copies (rRNA:rDNA ratios) between days 1 and 3 of the mesocosm incubations that was not observed in environmental isolates, which could indicate a different stress response. Nevertheless, there was no strong evidence of differential gene expression between environmental and enteric isolates related to habitat adaptation in the accompanying mesocosm metatranscriptomes. Overall, our results provide novel information on how rRNA levels may vary over different growth conditions (e.g., standard lab versus oligotrophic) for this important indicator bacteria. We also observed some evidence for habitat adaptation in E. faecalis; however, this adaptation may not be substantial or consistent enough for integration in water quality monitoring.

IMPORTANCE Enterococci are commonly used worldwide to monitor environmental fecal contamination and public health risk for waterborne diseases. However, closely related enterococci strains adapted to living in the extraenteric environment may represent a lower public health risk and confound water quality estimates. We developed an rRNA:rDNA viability assay for $E$. faecalis (a predominant species within this fecal group) and tested it against both enteric and environmental isolates in freshwater mesocosms to assess whether this approach can serve as a more sensitive water quality monitoring tool. We were unable to reliably distinguish the different isolate types using this assay under the conditions tested; thus, environmental strains should continue to be counted during routine water monitoring. However, this assay could be useful for distinguishing more recent (i.e., higher-risk) fecal pollution because rRNA levels significantly decreased after 1 week in all isolates.

KEYWORDS bioinformatics, environmental microbiology, fecal organisms, metatranscriptomics, public health, rRNA, water quality
Citation Suttner B, Kim M, Johnston ER Orellana LH, Ruiz-Perez CA, Rodriguez-R LM, Hatt JK, Brown J, Santo Domingo JW, Konstantinidis KT. 2021. Transcriptomic and rRNA:rDNA signatures of environmental versus enteric Enterococcus faecalis isolates under oligotrophic freshwater conditions. Microbiol Spectr 9:e00817-21. https://doi.org/10.1128/ Spectrum.00817-21.

Editor Jeffrey A. Gralnick, University of Minnesota

This is a work of the U.S. Government and is not subject to copyright protection in the United States. Foreign copyrights may apply. Address correspondence to Konstantinos T. Konstantinidis, kostas@ce.gatech.edu.

*Present address: Minjae Kim, Biosciences Division, Oak Ridge National Laboratory, Oak Ridge, Tennessee, USA.

${ }^{5}$ Present address: Eric R. Johnston, Biosciences Division, Oak Ridge National Laboratory, Oak Ridge, Tennessee, USA.

๑Present address: Luis H. Orellana, Max-PlanckInstitut für Marine Mikrobiologie, Bremen, Germany.

× Present address: Luis M. Rodriguez-R, Department of Microbiology and Digital Science Center (DiSC), University of Innsbruck, Innsbruck, Austria.

‡Present address: Joe Brown, Department of Environmental Sciences and Engineering, Gillings School of Global Public Health, University of North Carolina at Chapel Hill, Chapel Hill, North Carolina, USA.

Received 8 September 2021

Accepted 9 September 2021

Published 20 October 2021 
nterococci are used worldwide as fecal indicator bacteria based on the assumptions that they are predominantly found in intestinal systems of animal hosts and exhibit high die-off rates upon release to the natural environment. However, populations of Enterococcus spp. have been isolated from freshwater environments with no sign of recent fecal inputs (heretofore referred to as environmental strains) (1, 2). These environmental strains are phenotypically and phylogenetically indistinguishable from their enteric relatives based on standard selective media, so their recovery during a water quality test by conventional methods would be considered a positive indicator of fecal contamination $(3,4)$. Whole-genome comparisons of environmental and enteric strains have revealed distinct habitat-specific genetic signatures. For example, enteric genomes were specific or highly enriched for genes associated with antibiotic resistance, virulence, and the metabolism of sugars, while nickel and cobalt transport systems were overrepresented in the environmental genomes (4-6). These results suggested that the accessory gene content carried by different Enterococcus faecalis isolates may contribute to differential survival and adaptation in different habitats despite high genetic relatedness among core genes present in all isolates or the (shared) phenotypes in laboratory media $(4,6)$. However, the practical application and use of these alternative gene markers to distinguish innocuous environmental strains from enteric strains that indicate a risk to public health have not yet been tested. Moreover, elucidating the extent to which naturalized populations may confound water quality monitoring is critical for robust public health risk assessment.

Several studies have used cellular rRNA levels, often expressed as the copy number ratio of $16 \mathrm{~S}$ rRNA gene transcripts to $16 \mathrm{~S}$ rRNA gene DNA copies (rRNA:rDNA ratio), to detect active and/or growing microbes (7-10). This is based on the assumption that the levels of rRNA are much higher in actively growing and metabolizing cells than in dormant or dying cells. Further, examining the rRNA:rDNA ratio level may provide a more accurate assessment of the state of cellular activity than techniques based on membrane permeability (e.g., propidium monoazide quantitative PCR [PMA-qPCR] or live-dead staining microscopy) because cell death can occur before cell membrane lysis (11). Although the usefulness of the rRNA:rDNA ratio for these purposes has been documented for several bacterial genera, the relationship between rRNA:rDNA ratio and growth rate varies significantly between taxa, and some studies have even reported an inverse relationship between rRNA concentrations and growth rate (12-14). Since these ratios appear to be, at least partly, taxon specific, baseline data on rRNA:rDNA levels in enterococci during different stages of activity and decay are needed in order to determine if they can be used as a viability assay for water quality monitoring to distinguish environmentally adapted from enteric strains.

Accordingly, the guiding hypothesis of this study is that the strains associated with different habitats (i.e., enteric versus environmental) have distinct genetic and/or physiological adaptations that cause differential survival in freshwater ecosystems, and this can be detected and quantified for more accurate public health risk assessment based on rRNA:rDNA gene copy number ratios and whole-genome gene expression profiles. In particular, it is expected that environmentally adapted enterococci strains (if such strains exist) would have higher rRNA:rDNA ratios in surface water environments than enteric strains because the former strains are better able to survive environmental stressors like $\mathrm{O}_{2}$, sunlight, and nutrient limitation (15). In contrast, enteric strains, if they can persist in the same environment, are expected to be in a lower activity state.

To test this hypothesis, we performed laboratory mesocosm incubations that simulated the natural freshwater environment with three environmental and three enteric Enterococcus faecalis isolates that were previously reported to be phylogenetically and phenotypically indistinguishable from one another (4). The change in viable cell counts (i.e., plate counts) and rRNA:rDNA ratios were then monitored over 2 weeks to assess their decay and metabolic state. Therefore, this study provides important baseline information on rRNA levels in E. faecalis under different growth conditions and new insights into the use of rRNA as a target for improved water quality monitoring. We 
TABLE 1 E. faecalis isolates used in the dialysis bag mesocosm experiments ${ }^{a}$

\begin{tabular}{lll}
\hline Isolate name & Isolation source $^{b}$ & GenBank accession no. \\
\hline MMH594 & Enteric & AJDZ01000001.1 \\
ERV62 & Enteric & ALZQ01000001.1 \\
TX0104 & Enteric & ACGL01000001.1 \\
MTUP9 & Environmental & AYOJ01000001.1 \\
MTmid8 & Environmental & AYKU01000001.1 \\
AZ19 & Environmental & AYLU01000001.1 \\
\hline
\end{tabular}

aTotal RNA from the mesocosm samples was also analyzed with metatranscriptomics for the isolates in bold. bIsolation source describes whether the strains were isolated from the human gut (enteric) or freshwaters with unknown history of fecal pollution sources (environmental) according to the method of Weigand et al. (4).

focused on $E$. faecalis because it is a prominent member of the enterococci group that includes some of the best-characterized environmental and enteric isolates with genomic techniques (for an example, see reference 4).

\section{RESULTS}

E. faecalis strains used in the mesocosm incubations. Individual E. faecalis isolates ( 3 enteric and 3 environmental [Table 1]) were selected based on previous comparative genomic analysis that showed that these isolates contain putative habitat-specific gene signatures (4) that may contribute to differential survival under oligotrophic conditions. The environmental isolates were recovered from geographically disparate watersheds using standard selective medium for enumerating enterococci. Although the isolation sites may be impacted by nearby wastewater treatment plants or concentrated animal feeding operations, there were no signs of fecal contamination at the time of sampling (see details in the work of Weigand et al. [4]). The enteric isolates were publicly available with detailed human body site isolation information and carried the sugar utilization genes thought to be indicative of adaptation to the gut (4). All of these six strains shared $>97 \%$ average nucleotide identity (ANI), well above the 95\% ANI cutoff used for species demarcation (16). Further details on the source and genome content of these isolates are described in the work of Weigand et al. (4).

rRNA:rDNA ratios of enteric versus environmental $E$. faecalis isolates in dialysis bag mesocosms simulating an oligotrophic freshwater habitat. In our pilot experiment using glass bottle mesocosms to simulate oligotrophic lake water conditions, enteric and environmental strains of $E$. faecalis showed similar decreases in viable cell counts over time, while qPCR counts remained constant (see the supplemental material for details). However, in this pilot experiment using only traditional qPCR of the 16S rRNA gene, we were unable to measure any differences in cellular viability (i.e., live or dead) between the two strain types using these methods (Fig. S1). Therefore, we subsequently developed and used an rRNA:rDNA ratio assay to compare relative activity levels in human enteric versus environmental $E$. faecalis isolates in laboratory dialysis bag mesocosms simulating an oligotrophic freshwater environment. Specifically, $E$. faecalis isolates were spiked in known concentrations in filter-sterilized lake water that was enclosed in dialysis bags, and the bags were subsequently incubated in 10-gal aquarium tanks filled with (unfiltered) lake water. Dialysis bags allow water and nutrients to pass through but not cells and thus represent convenient systems for incubations that simulate well in situ conditions. Mesocosm sampling occurred on days 1, 3, 8, and 11 and included plating for viable cell counts and filtering for total nucleic acid extraction to determine rRNA:rDNA ratios over time as a general proxy to compare relative levels of cellular activity between the two strain types. Although the lake water used in the dialysis bags was filter sterilized before inoculation (i.e., to remove predators), some growth was observed in water from the negative-control bags by day 8 on tryptic soy agar (TSA) plates. However, no growth was observed on the Enterococcus-specific media (data not shown). This result suggested that the integrity of the dialysis bags started to break down over time and that some of the microbes from the nonsterilized lake water in the tanks outside the bags were 

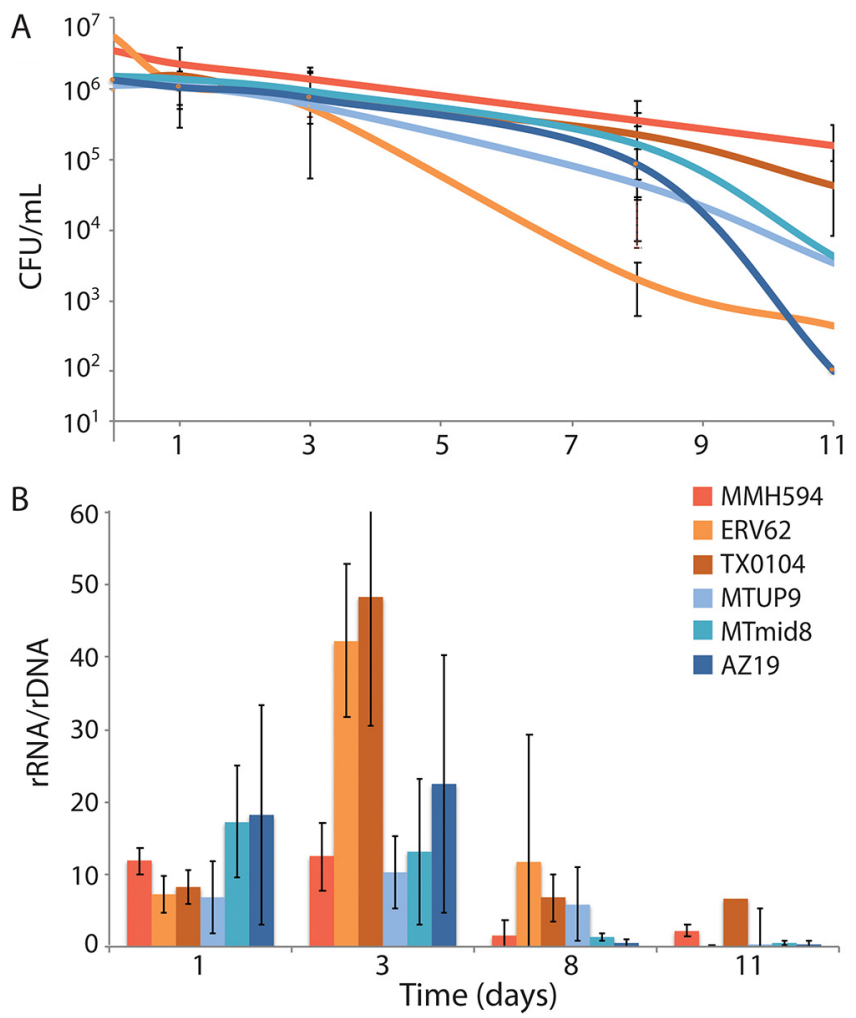

FIG 1 Comparison of changes in viable cell counts (A) and rRNA:rDNA ratios (B) of enteric versus environmental $E$. faecalis isolates over time in dialysis bag mesocosms. Three enteric and three environmental isolates are represented by different shades of orange and blue, respectively. Error bars are standard deviations among three technical replicates.

able to pass through the dialysis membranes. Hence, we primarily focused our analysis and interrelations on the first three sampling points.

All E. faecalis strains exhibited a decrease in viable cell and 165 qPCR counts for the duration of the experiment (i.e., until day 11). Moreover, decay rates based on plate counts were not significantly different between the two isolate types, i.e., environmental versus enteric (paired Wilcoxon, $P=0.063$ ), consistent with our previous pilot experiment (Fig. 1A and Fig. S1). The average rRNA:rDNA ratios in the three environmental and one of the enteric isolates were relatively stable from days 1 to 3 (0.7- to 1.5-fold change in the ratio). However, two of the enteric strains (ERV62 and MMH594) had an $\sim 6$-fold increase in their rRNA:rDNA ratios from days 1 to 3 (Fig. 1B). By days 8 and 11 , the average ratios decreased and approached zero consistently for all six isolates. When comparing average rRNA:rDNA ratios overall (i.e., enteric versus environmental across all time points), it was found that the enteric isolates were not significantly different from environmental isolates (Wilcoxon rank sum, $P=0.149$ ). When looking at the habitat types separately (i.e., among themselves) over time, the average rRNA:rDNA ratios between day 1 and day 3 were not found to be significantly different for the three environmental strains but were significant for the three enteric strains (paired Wilcoxon, $P=1.0$ and 0.014 , respectively). This result suggested that the enteric and environmental isolates may show different gene expression responses to environmental stress (e.g., nutrient limitation), which we examined more fully with metatranscriptomics.

Comparative metatranscriptomics of enteric and environmental isolates. The 16S rRNA:rDNA ratio alone does not provide information about specific gene functions and differences in mRNA expression levels that may serve as more sensitive biomarkers for the response to oligotrophic freshwater conditions. Thus, we used metatranscriptome sequencing profiles of the dialysis bag mesocosms to identify specific metabolic pathways that may underlie habitat adaptation and represent more reliable targets for 
improved fecal indicator bacterium (FIB) assays. Due to cost considerations and the number of samples with sufficient RNA for transcriptome sequencing (RNA-seq) library preparation, we selected a subset of the mesocosm samples (two environmental and two enteric strains [Table 1]) for total community RNA sequencing with an internal spiked control for absolute transcript quantification. The two enteric isolates were also selected because they showed the most dramatic increase in rRNA:rDNA ratio between day 1 and day 3. Because the original RNA extraction protocol used was designed for rRNA:rDNA analysis and was not optimized for mRNA sequencing (i.e., depletion of 16S rRNA gene transcripts), we were not able to get enough total RNA for rRNA-subtracted libraries. Thus, total RNA was sequenced instead. The resulting metatranscriptome libraries yielded an average of $3.2 \times 10^{7}\left( \pm 9.7 \times 10^{6}\right)$ reads per sample, and $\sim 95.7 \%$ of those reads were rRNA. The internal RNA standard recovery in each metatranscriptome ranged from $0.02 \%$ to $0.13 \%$ of the original spike-in quantity, as originally planned. The internal standard percent recovery was used to estimate the absolute number of mRNA reads per nanogram of RNA sequenced $\left(5.9 \times 10^{7} \pm 3.5 \times 10^{7}\right.$ on average in each sample) following the methods described by Satinsky et al. (17).

Reference genome sequences of the isolates were previously determined (Table 1) and were used for read mapping to identify genes with significantly different expression between the two isolate types. Overall, there were no differentially expressed genes (DEGs) between enteric and environmental isolates across all time points. Furthermore, since increasing contamination was observed over time as the dialysis bags started to break down (see above), we restricted our DEG analysis to include only the day 1 and 3 time points. When controlling for the effect of time, there were only 31 strain-specific DEGs with adjusted $P$ values $\left(P_{\text {adj }}\right)$ of $<0.05$ observed between day 1 and day 3, with 24 and 8 genes being more expressed in the environmental and enteric isolates, respectively (Fig. 2). None of these genes were among the habitat-specific genes identified by the previous comparative genomic studies, such as the nickel uptake operon, nik(MN)QO (4-6). The DEGs found in the environmental isolates were mostly housekeeping genes such as the genes for ribosomal and transcription-related proteins (e.g., tRNA ligase and elongation factor T [Fig. 2]). In contrast, genes potentially related to cellular stress response, such as a putative transcription repressor gene (niaR), a DNA replication and repair gene (recF), and a zinc-transporting ATPase gene (zosA), had higher expression in the enteric isolates. Metal ions, such as $\mathrm{Zn}^{2+}, \mathrm{Cu}^{2+}$, and $\mathrm{Mn}^{2+}$, are known to be important for oxidative stress defense in commensal $E$. faecalis (18). Together these results suggest that a few genes expressed differentially among the strains may be linked to different habitat adaptation, but the great majority of genes in the genome did not show differential gene expression.

rRNA:rDNA ratios over the standard growth curve in pure culture. Since the relationship between rRNA:rDNA ratios and growth rate is taxon specific (11), we also collected baseline data on rRNA:rDNA levels in pure cultures of $E$. faecalis under standard laboratory conditions, which has not been examined previously for this species. These data were collected to compare against the ratios observed under oligotrophic conditions in our laboratory dialysis bag mesocosms. A typical bacterium growth curve was observed in these pure culture experiments, in which the exponential growth phase lasted $\sim 10 \mathrm{~h}$ and maximum cell density $\left(1.5 \times 10^{9} \mathrm{CFU} / \mathrm{ml}\right)$ was observed at $12.5 \mathrm{~h}$ (Fig. 3). Cell density remained relatively stable until the next measurement at $25 \mathrm{~h}$, at which time cell density was still around $1.1 \times 10^{9} \mathrm{CFU} / \mathrm{ml}$ (Fig. 3). The rRNA:rDNA ratios ranged from 5.5 to 372 , with the lowest ratios being observed during early exponential growth phase (i.e., during the first $5 \mathrm{~h}$ ), after which point the ratio started to increase but there was a high level of variation between biological replicates (Fig. 3). The highest levels of rRNA:rDNA ratios were observed in the early stationary phase $(\sim$ hour 12 ; average ratio $=372)$, after which the ratios started to decrease during stationary and death phases.

\section{DISCUSSION}

This study investigated whether the rRNA:rDNA ratio can be used to distinguish enteric versus environmental strains of $E$. faecalis for improved environmental water 


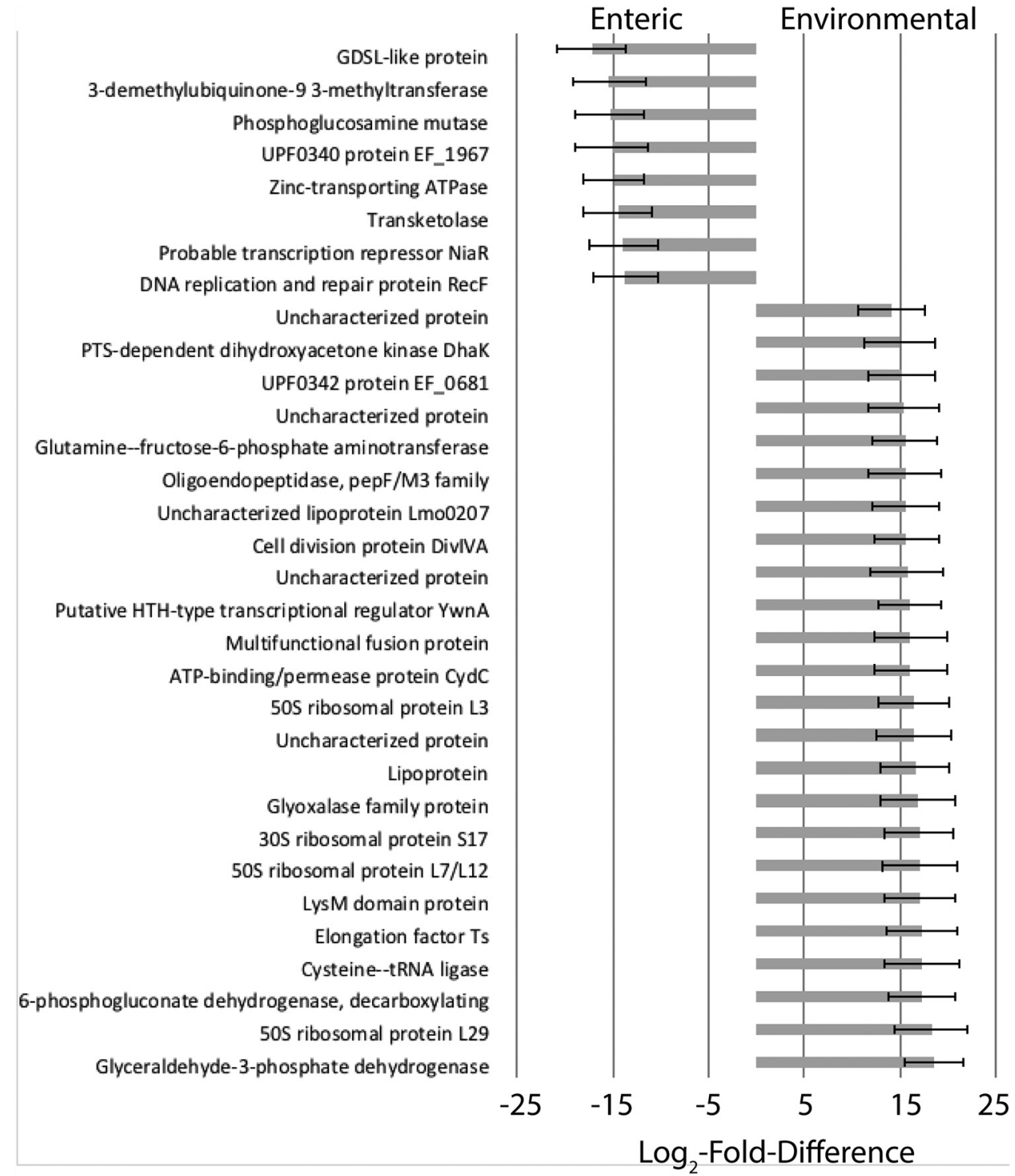

FIG 2 Differentially expressed genes between enteric and environmental $E$. faecalis isolates between days 1 and 3. Shown are functional genes that were significantly more expressed $\left(P_{\text {adj }}<0.05\right)$ in enteric relative to the environmental isolates (negative $\log _{2}$ fold difference) or significantly more expressed in environmental relative to enteric isolates (positive $\log _{2}$ fold difference). Error bars represent the standard errors among biological replicates. None of the putative, habitat-specific auxiliary genes were significantly differentially abundant, and thus, they are not shown here.

quality monitoring (15). We observed high variability in rRNA:rDNA ratios among biological replicates for all strains under oligotrophic mesocosm growth conditions (Fig. 1B). Notably, the ratios under these conditions were, on average, roughly 2 orders of magnitude lower than those observed under standard lab conditions in pure culture (Fig. 3). These results are consistent with those of another study, which showed a high standard deviation in ratios and that copiotrophs have much lower ratios during growth under oligotrophic conditions relative to growth in rich media (19). Therefore, it appears that the rRNA:rDNA ratio could reflect oligo- versus copiotrophic growth conditions for $E$. faecalis.

Although there was a difference between rRNA:rDNA ratios observed in the enteric and environmental isolates on day 3 (Fig. 1B), our results were not conclusive with respect to whether this assay is suitable for distinguishing isolate types in water quality monitoring applications because the differences were not large enough and were 


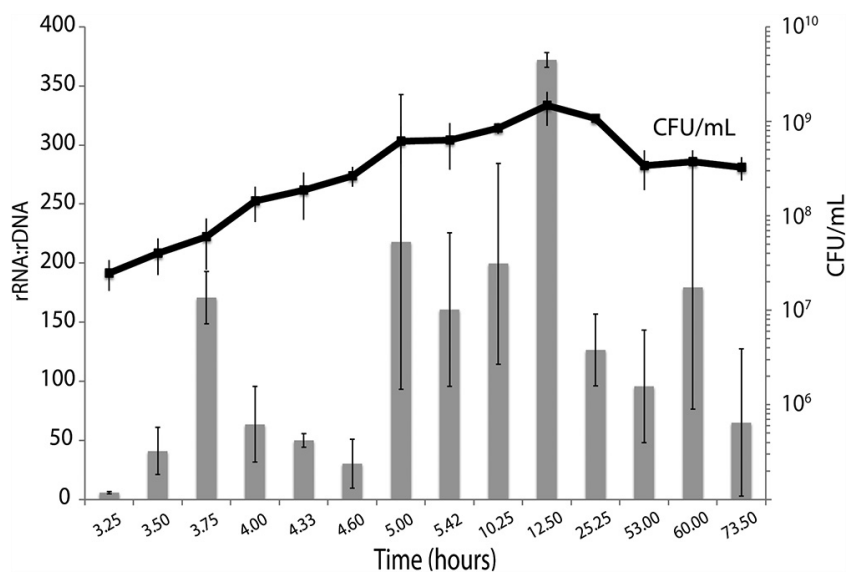

FIG 3 Cellular abundance and rRNA:rDNA ratios for E. faecalis MTUP9 (an environmental isolate) under standard pure culture conditions. The same strain was grown in three separate batch cultures (biological replicates) and at each time point, each biological replicate was sampled 3 times (technical replicates) for nine total measurements at each time point. These nine measurements were averaged, and error bars are standard deviations of biological and technical replicates.

strain specific (as opposed to habitat type specific), at least for the conditions tested in this study. However, the rRNA:rDNA ratio may be useful in pinpointing the age of a fecal pollution incident. All six isolates had significantly higher rRNA:rDNA ratios on days 1 and 3 compared to days 8 and 11, with overall average ratios of 11.7, 24.8, 4.7, and 1.8 , respectively (Fig. 1B). That is, the rRNA:rDNA ratio was substantially higher in the early stages, and this could serve as a sign of recent fecal pollution. Specifically, higher ratios $(\sim 12$; based on the median rRNA:rDNA ratios observed for all strains on days 1 and 3) could indicate a more recent pollution event, whereas lower ratios (e.g., $<1.5$; based on the median ratios observed for all strains on days 8 and 11) could indicate that the public health risks from exposure to pathogens are not as high. The rRNA: rDNA can also reflect the physiological status of the group of organisms targeted, as a whole, in environmental waters (15) and thus may also be explored further as a tool for determining favorable conditions for persistence and regrowth from the viablebut-not-culturable (VBNC) state (20).

The viable cell counts indicated that the abundance of $E$. faecalis organisms still exceeded the Environmental Protection Agency (EPA) recreational water quality criteria of fewer than 35 colony forming units (or CFUs) per $100 \mathrm{ml}$ in any 90-day interval for all isolates on day $8\left(\sim 10^{5} \mathrm{CFU} / \mathrm{ml}[\right.$ Fig. $\left.1 \mathrm{~A}]\right)$; thus, these lake water samples would still be considered a public health risk according to current EPA standards (21). Total E. faecalis cell density estimates based on plate counts exceeded those based on 16S qPCR in all isolates on days 1 and 3, but this trend started to reverse by day 8 in most of the strains (Table 2). The discrepancy between plate counts and qPCR is most likely the result of biomass lost during simultaneous DNA and RNA extraction from the filters. Furthermore, our finding that the rRNA:rDNA ratio decreases after day 3 suggests that these cells have largely become inactive (e.g., enter VBNC) and/or have started dying; hence, they represent a lower risk than cells at day 1 . Consistent with these interpretations, a recent quantitative microbial risk assessment (QMRA) analysis of sewage pollution suggested that the risk of exposure to pathogens is not significant after 3 days (22). In water bodies that consistently exceed EPA regulations for enterococci, it could be useful to investigate whether this is the result of a natural reservoir (i.e., no pathogen risk) or chronic pollution (pathogen risk), and techniques like the rRNA:rDNA assay presented here could be useful to help inform appropriate monitoring, management, and/ or mitigation strategies. Further studies are needed to more fully characterize the changes in the rRNA:rDNA ratio during different growth states of E. faecalis that are relevant to public health and water quality monitoring, such as the VBNC (23-25) versus dying states. 
TABLE 2 Ratio of viable plate counts to qPCR-based cell density estimates in the dialysis bag mesocosm experiments ${ }^{a}$

\begin{tabular}{lllll}
\hline & \multicolumn{3}{l}{ Value for: } \\
\cline { 2 - 5 } Isolate & Day 1 & Day 3 & Day 8 & Day \\
\hline MMH594 & 4.45 & 5.57 & 3.47 & 4.54 \\
ERV62 & 4.18 & 5.85 & 0.07 & 0.29 \\
TX0104 & 2.91 & 5.26 & 1.54 & 3.36 \\
MTUP9 & 2.59 & 3.19 & 1.07 & 0.74 \\
MTmid8 & 6.41 & 7.77 & 0.56 & 0.41 \\
AZ19 & 5.42 & 3.68 & 0.80 & 0.002 \\
\hline
\end{tabular}

aThe $16 \mathrm{~S}$ rRNA gene qPCR-based count estimates were converted to cells per milliliter assuming four copies of the rRNA gene operon per genome.

Notably, two of the three enteric isolates showed a 6-fold increase in their rRNA: rDNA ratios from days 1 to 3 (Fig. 1B), and the ratios on day 3 for these two isolates ( $\sim 5$ rRNA:rDNA) were similar to the lower end of average values observed for $E$. faecalis in pure culture (e.g., during early exponential phase [Fig. 3]). However, our results based on viable cell or PCR counts suggested that $E$. faecalis isolates were not actively growing or replicating in the mesocosms over time. Moreover, the incubation conditions are remarkably different between oligotrophic growth and growth in pure culture; hence, the trends observed in the two experiments (i.e., lake water mesocosm versus pure batch culture) are presumably the result of different biological factors.

A potential explanation for increasing rRNA:rDNA ratios coupled to the decreasing cell counts observed under the oligotrophic mesocosm conditions is that the enteric isolates increase gene expression for pathways related to nongrowth activities, such as environmental stress or cell homeostasis that results in more ribosomes (and thus more rRNA). Accumulating or maintaining high rRNA levels during periods of low activity may confer a competitive advantage upon return to favorable conditions, especially in copiotrophic environments that favor fast growers that can respond quickly to nutrient stimuli (26). Enteric isolates maintaining high cellular rRNA levels through day 3 could indicate an adaptive strategy for high nutrient environments like the gut, whereas the environmental isolates are not "evolutionarily primed" to expect high nutrient influxes and do not devote as much energy to maintaining high rRNA levels.

Consistent with these interpretations, metatranscriptomics analysis of the mesocosm incubation samples revealed that the enteric isolates differentially expressed several genes (DEGs) whose functions potentially reflect a stronger stress response compared to environmental isolates. This conclusion is also supported by a recent study that showed soil microbes adapted to low-phosphorus conditions had much higher transcription of housekeeping genes under phosphorus limitation (27). In contrast, DEGs of environmental strains included only a few housekeeping genes such as ribosomal and transcription-related proteins (Fig. 2), which may indicate better survival because they are able to maintain general gene expression without a strong signal of environmental stress. However, the sample size and number of DEGs overall was small (only 31 DEGs in total, and 30 of these had $P_{\text {adj }}$ values of $>0.031$ ) and these results may be spurious, as about half of the DEGs detected could be due to chance based on the false-discovery rate predicted by the DESeq 2 analysis (i.e., expected $\sim 17$ DEGs by chance). Therefore, although our results provided some evidence that environmental and enteric isolates may respond differently upon release to the natural environment, the differences observed were too small and/or not consistent enough to provide robust means to distinguish between these two groups of isolates, at least for the conditions simulated by our mesocosm. It is possible that the environmental isolates tested in this study are not truly adapted to grow in the extraenteric environment or-at least-the specific conditions of our mesocosms (e.g., they quickly died out during our mesocosm) and this accounted, partly, for the overall small differences observed with enteric isolates. However, the environmental isolates were obtained during regular 
monitoring of watersheds using the established EPA methods (4); thus, our results are relevant for microbial water quality applications, in any case.

Previous studies of other copiotrophs under balanced growth conditions in pure culture have shown that cellular rRNA concentration correlates well with growth rate $(8$, $28,29)$. Therefore, we expected to see the highest rRNA:rDNA ratios for $E$. faecalis during the exponential phase in pure culture. However, the highest ratios were observed around hour 12, when growth was entering early stationary phase (Fig. 3). The relationship between RNA levels and growth is not linear or consistent between different taxa, especially in environmental oligotrophic bacteria $(13,30)$. Therefore, this result is not necessarily surprising, but it does suggest that the regulation of cellular RNA levels in environmental isolates of $E$. faecalis may be more complicated and not linearly correlated with growth. One possible explanation for the trends observed is that during early exponential phase, the cells are rapidly replicating their genomes and may have multiple genome copies per cell during rolling replication, resulting in the observed lower rRNA:rDNA ratios. As nutrients in the batch culture start to become depleted and cell growth slows, there is a lag in the ribosome transcription feedback loop around hour 12 , at which time the ribosome concentration briefly exceeds cell demand for rapid growth and results in the observed higher ratio. The high variation between biological replicates observed in both experiments also suggests that this rRNA:rDNA assay should be tested in more isolates in order to confirm the preliminary trends reported herein and the amount of natural variability in rRNA:rDNA ratios between isolates as well as to provide more support for the explanations proposed above.

Furthermore, we acknowledge that the growth conditions employed in this study may also limit our ability to distinguish isolates from the two habitat types. Previous starvation experiments showed that in some taxa, growing cells at maximum or medium growth rates before starvation can affect whether high rRNA levels are sustained even when cell activity decreases $(31,32)$ and suggested that an organism's response to an event (e.g., introduction to an extraenteric environment through fecal shedding) can be determined by the existing conditions before that event. In our dialysis bag mesocosm experiment, we spiked pure cultures grown in rich media into lake water, which may not accurately reflect the life histories of environmental or enteric $E$. faecalis isolates, and thus, different ratios may be observed in situ relative to our mesocosm condition. For example, an enteric cell is likely first introduced into a sewage or septic system which may not be nutrient limiting but have other stressors, like oxidation or predation, before reaching a surface water body. It is also possible that the habitat-specific genes previously identified, such as those encoding the nickel and cobalt transport systems in the environmental genomes (4-6), are tuned for different conditions or stimuli rather than the mesocosm conditions used in this study, and this accounts for the lack of differential expression of these genes in our data sets. Although mesocosm studies are helpful for comparing $E$. faecalis survival in a more controlled environment, they cannot simulate all of the complex biotic and abiotic factors that occur in aquatic habitats. Inspecting the ratios in extractions directly from known, natural extraenteric reservoirs of Enterococcus, such as in algal mats (33), could help to get a better understanding of how rRNA levels are regulated in isolates that have been (presumably) under nutrient limitation for a longer period. For example, E. faecalis rRNA:rDNA ratios observed in water samples after a combined sewer overflow (CSO) event were $\sim 1.85$ (15), which is similar to the ratios observed on day 11 in this study, but it is not clear how long the CSO E. Faecalis populations were exposed to the extraenteric environment and how this rRNA:rDNA ratio relates to public health risks.

Finally, the RNA extraction protocol used in this experiment was originally optimized for the rRNA:rDNA assay (i.e., simultaneous and consistent extraction of both DNA and RNA from a single filter to ensure that the same amount of starting material is used for both $\mathrm{qPCR}$ and reverse transcriptase quantitative PCR [RT-qPCR]) and resulted in extractions with total RNA concentrations too low for rRNA-subtracted libraries. Accordingly, our metatranscriptomics data sets included a minority of reads 
representing protein-coding genes ( $<5 \%$ mRNA; typical for non-rRNA-subtracted libraries), and some of the DEG signal could have been lost as a result of this (i.e., only the most highly expressed transcripts were detected in the metatranscriptomes due to low sequencing coverage of mRNAs overall). It should be mentioned, however, that cDNA reads covered the whole $E$. faecalis references genomes at $9 \times$, on average, for day 1 ; thus, we should have been able to detect most DEGs on day 1, and without any possible biases introduced by an rRNA gene depletion step (since rRNA gene depletion protocols were not applied here). At later time points, when the E. faecalis metatranscriptome signal was decreasing, consistent with the decreasing viable cell counts, we were able to detect only highly expressed genes as DE based on an $\sim 2 \times$ coverage of the genome, on average, by cDNA reads. Thus, even though a few truly differentially expressed genes may have escaped detection for the reasons mentioned above, the overall small differences observed in the transcriptomes of enteric versus environmental isolates represent a robust result. Future studies should include separate RNA extractions for the rRNA:rDNA ratio assay and metatranscriptomic sequencing with possibly rRNA (ribo)-subtracted libraries.

Despite these limitations, our work provides useful information on rRNA:rDNA ratios in $E$. faecalis under both standard lab and in situ-like conditions relevant for environmental water quality monitoring, which has only been investigated in natural water samples for this genus $(15,34)$. Our results provide some evidence for different habitat adaptations between environmental and enteric strains, but the difference may be too subtle or not consistent enough to be used in water quality monitoring. Further, working with RNA is generally more difficult and expensive than working with DNA (e.g., often requires a $-80^{\circ} \mathrm{C}$ freezer, RNase-free consumables, etc.) and requires more technical expertise and higher sterility, making this approach impractical for local municipalities or regulatory agencies with limited laboratory resources. That said, our study showed that the rRNA:rDNA ratio may be useful for determining more recent fecal versus older pollution events. Furthermore, our results provided new insights on the relationship between rRNA levels and nongrowth activities for an important FIB taxon. Evaluating more strains and growth conditions would be necessary to confirm our preliminary findings and establish whether rRNA:rDNA-based methods can provide more robust public health risk assessments.

\section{MATERIALS AND METHODS}

Comparison of rRNA:rDNA ratios of enteric versus environmental E. faecalis isolates in dialysis bag mesocosms. (i) Dialysis bag mesocosm setup. Lake water was collected from Lake Lanier (Georgia, USA; $34^{\circ} 15^{\prime} 38.898^{\prime \prime} \mathrm{N}, 83^{\circ} 56^{\prime} 56.0328^{\prime \prime} \mathrm{W}$ ) in June 2017 using acid-washed 10-liter carboys, transported immediately back to the lab, and stored at $4^{\circ} \mathrm{C}$ for mesocosm setup the following day. Lake water used for inoculation with the E. faecalis isolates was first filtered through $0.2-\mu \mathrm{m}$ Sterivex filters as described previously (35) to remove predation pressure. The remaining unfiltered water was used to fill 10-gal aquarium tanks in which dialysis bags were suspended during the incubations, as described below. Frozen glycerol stocks of the $E$. faecalis isolates (Table 1 ) were streaked for single colonies onto tryptic soy agar (TSA) plates and grown overnight at $37^{\circ} \mathrm{C}$. A single colony from each isolate was then inoculated into $4 \mathrm{ml}$ of tryptic soy broth (TSB) and incubated at $37^{\circ} \mathrm{C}$ with shaking at $150 \mathrm{rpm}$ for $14 \mathrm{~h}$. One milliliter from each overnight culture was washed once with phosphate-buffered saline (PBS) before inoculation into filtered lake water to a final concentration of $\sim 10^{6} \mathrm{CFU} / \mathrm{ml}$. The initial concentration for each overnight culture was also determined by plate counts on TSA plates. The dialysis bags (6- to 8-kDa-molecularweight cutoff) were filled to a total volume of $110 \mathrm{ml}(\sim 21-\mathrm{cm}$ length of 32-mm-diameter dialysis tubing) and closed on both ends using polypropylene Spectra/Por clamps (Spectrum Laboratories). The dialysis bags have a pore size that allows passage of small molecules and ions but prevents passage of bacterial cells and viral particles. Enough dialysis bags were filled to sample each isolate in triplicate at four time points, plus four filtered lake water negative-control bags. The dialysis bags were then transferred to 10-gal aquarium tanks filled with unfiltered lake water and stored in environmentally controlled rooms at $22^{\circ} \mathrm{C}$ in the dark. A small water pump was included in each tank for aeration and nutrient distribution. A small headspace of air was left in each bag when sealing with the clamps so that they could float freely in the tanks.

(ii) Mesocosm sampling. Each sampling time point included triplicate biological replicates per isolate and a single lake water negative control. Destructive sampling of the dialysis bags occurred at days $1,3,8$, and 11 after the initial setup. Fifty milliliters from each dialysis bag was filtered through $0.45-\mu \mathrm{m}$ polycarbonate membranes, and then the filters were transferred to 2-ml screw-cap tubes that had been prefilled with $0.8 \mathrm{ml}$ of Qiagen buffer RLT (with 1\% beta-mercaptoethanol) and $100 \mathrm{mg}$ of acid-washed 0.1-mm glass beads. Bead tubes were stored at $-80^{\circ} \mathrm{C}$ until ready for extraction (storage time was $<1$ month for all filters). Additionally, water from each bag was 10-fold serially diluted with PBS for culture-based enumeration on TSA and 
mEnterococcus agar (BD Difco) plates. All dilutions yielding measurements within the acceptable range of quantification were averaged to estimate CFU per milliliter of each isolate. The filtered lake water was also checked for sterility by plating on TSA and mEnterococcus agar at day 0 before inoculating with $E$. faecalis isolates.

(iii) Total nucleic acid extraction. The frozen filters were defrosted on ice before the cells were mechanically lysed using a BioSpec Mini-BeadBeater-24 in four 1-min intervals with icing in between to prevent the samples from excessive heating and to protect the integrity of the RNA. Total nucleic acids were extracted from cell lysates using the Qiagen AllPrep DNA/RNA miniextraction kit following the manufacturer's protocol for animal tissue. Contaminating DNA was removed from RNA samples by digestion (1 or 2 times depending on the sample concentration) with the Ambion TURBO DNase kit following the manufacturer's protocol. RNA integrity was assessed with an Agilent 2100 Bioanalyzer instrument and the Agilent RNA 6000 Pico kit. RNA samples used for downstream analysis generally had an RNA identification number (RIN) of $>7$ and a 23S/16S rRNA ratio of $>1$ based on the Agilent 2100 instrument.

(iv) Assessment of the quality of the RNA extractions. Elimination of DNA from RNA samples was confirmed by endpoint PCR amplification with the same primers as used for the E. faecalis-specific 16S rRNA qPCR assay (36). Two microliters of undiluted RNA was used as the template in 20- $\mu$ l PCRs with $0.5 \mu \mathrm{M}$ primers, $200 \mu \mathrm{M}$ deoxynucleoside triphosphates (dNTPs), $0.5 \mathrm{U}$ of TaKaRa Ex Taq polymerase, and $1 \times$ TaKaRa PCR buffer. The thermocycling conditions were as follows: $1 \mathrm{~min}$ at $95^{\circ} \mathrm{C}$ and then 30 cycles of $95^{\circ} \mathrm{C}$ for $15 \mathrm{~s}$ and $61^{\circ} \mathrm{C}$ for $30 \mathrm{~s}$ followed by $72^{\circ} \mathrm{C}$ for $1 \mathrm{~min}$. The PCR products were visualized with gel electrophoresis, and the absence of any detectable bands in the gel indicated that there was no significant DNA contamination in the RNA samples.

The absence of PCR inhibitors in the RNA samples was confirmed by endpoint PCR amplification in which a known amount ( $\sim 10^{7}$ copies) of standard plasmid DNA was spiked into a PCR in the presence of RNA. The same PCR master mix and thermocycling conditions as described above were used except the primers targeted the nickel uptake gene in the standard plasmid (not published). If the RNA preparation contained inhibitors, amplification of the DNA template was expected to be inhibited in the presence of RNA. The PCR products were run on a $1 \%$ agarose gel, and the presence of a single band at the expected size and yield of the PCR amplicon from the standard plasmid template confirmed the absence of any PCR inhibitors.

(v) Quantification of 165 rRNA and rDNA using RT-qPCR and qPCR. DNA and RNA concentrations were quantified using the Qubit high-sensitivity (HS) DNA and RNA kits (Thermo Fisher Scientific), respectively, and the Qubit 2.0 fluorometer. Template nucleic acids were then diluted to below $0.5 \mathrm{ng} / \mu \mathrm{l}$ before amplification using an E. faecalis-specific 16S rRNA gene assay (36). The standard plasmid used for absolute quantification was a full-length $E$. faecalis 16S rRNA gene ligated into the pCR2.1-TOPO TA vector and cloned into One Shot chemically competent TOP10 Escherichia coli using the TOPO-TA cloning kit (Invitrogen) following the manufacturer's instructions. Eight 10-fold serial dilutions ( $10^{8}$ to $10^{1}$ copies per reaction) of quantitative PCR ( $q P C R$ ) standard plasmids were assayed in triplicate on every 96-well plate for absolute quantification. All reactions were performed on the Applied Biosystems 7500Fast machine using Bio-Rad iTaq universal probe one-step reagents following the manufacturer's protocol. Reactions were performed in triplicate in a total volume of $20 \mu \mathrm{l}$ that included $2 \mu \mathrm{l}$ of the template or standard plasmid and $250 \mathrm{nM}$ each primer and TaqMan ( 5 ' hydrolysis) probe (the reverse transcriptase quantitative PCRs [RT-qPCRs] also included $0.5 \mu \mathrm{l}$ of Bio-Rad iScript advanced reverse transcriptase). Thermocycling conditions for qPCR consisted of an initial $50^{\circ} \mathrm{C}$ step for $2 \mathrm{~min}$ followed by $95^{\circ} \mathrm{C}$ for $10 \mathrm{~min}$ and then 40 cycles of $95^{\circ} \mathrm{C}$ for $15 \mathrm{~s}$ and $60^{\circ} \mathrm{C}$ for $60 \mathrm{~s}$. The RT-qPCR thermocycling conditions were the same except for the initial step of $50^{\circ} \mathrm{C}$ for $10 \mathrm{~min}$ followed by $95^{\circ} \mathrm{C}$ for $2 \mathrm{~min}$. The calibration curve from each plate was used to calculate rRNA and rDNA copy numbers in each sample, which were averaged among technical replicates, multiplied by the elution volume (200 or $50 \mu$ l for DNA or RNA, respectively), and then divided by the filter volume $(50 \mathrm{ml})$ to give total copies per milliliter of mesocosm water sampled.

(vi) Statistical analyses. Culture-based cell counts over time were averaged by habitat type and tested for significant difference between the two groups using the paired Wilcoxon signed-rank test in base R. The rRNA:rDNA ratios of each isolate were compared by habitat type overall and between each time point. Since the data violated the Bartlett test of equal variance (as implemented in base R), nonparametric pairwise multiple comparisons were performed using the Wilcoxon signed-rank test with the Holm $P$ value correction for multiple comparisons using a custom R function (http://www.statmethods.net/RiA/wmc.txt).

Metatranscriptome sequencing and analysis of total RNA from dialysis bag mesocosms. (i) Metatranscriptome library preparation and sequencing. Triplicate RNA extractions from each isolate at each of the four time points were pooled in order to obtain enough high-quality RNA for metatranscriptomic sequencing., and cDNA libraries were prepared using the ScriptSeq v2 RNA-seq library preparation kit (Illumina) following the manufacturer's instructions except that a half nanogram $(\sim 1 \%$ of total library size) of a luciferase internal RNA standard was included during the RNA fragmentation (step 3A) for absolute quantification of transcript copy numbers as described below. The quality and insert size of each cDNA library were determined using the Agilent HS DNA kit and Agilent 2100 Bioanalyzer instrument. Library concentrations were determined using the Qubit HS DNA kit before pooling and loading into the flow cell according to the manufacturer's recommendations and sequencing on the Illumina HiSeq 2500 instrument as described previously (27).

(ii) Luciferase internal RNA standard preparation. The Promega pGEM-luc plasmid vector (GenBank accession number X65316) containing a 1,094-nucleotide fragment of the firefly luciferase gene was digested with Sphl-HF restriction enzyme (New England BioLabs) at $37^{\circ} \mathrm{C}$ for $1 \mathrm{~h}$ to linearize the plasmid, followed by the Qiagen PCR cleanup kit to stop the reaction. The digested DNA was gel purified using a 1.5\% low-melting-point agarose gel and the MO BIO UltraClean 15 DNA purification kit followed by end repair with the Thermo Scientific fast DNA end repair kit and another cleanup with the Qiagen PCR cleanup kit but with a 30- $\mu$ l elution volume. The DNA was concentrated by ethanol precipitation before 
transcribing to RNA with the Promega Riboprobe in vitro transcription T7 system and following the manufacturer's protocol 4.F for synthesis of large amounts of RNA. The RNA standard quantity and quality were determined using the Qubit HS RNA kit and Agilent Bioanalyzer as described above.

(iii) Transcriptome sequence analysis. All transcriptomic reads were quality filtered and trimmed as described previously (37). Trimmed reads were filtered to remove rRNA sequences using SortMeRNA v2.1 (38) with all rRNA databases in the program and the following options: -blast 1 -num_alignments 1 $-\mathrm{v}-\mathrm{m}$ 8336. The internal luciferase standard sequences were identified by blastn search against the 1,094-bp nucleotide luciferase reference sequence carried on the pGEM-luc plasmid vector (GenBank accession number X65316). Luciferase matches were filtered for best match using a threshold of $97 \%$ identity and alignment length that is $80 \%$ of the query read length, and resulting matches were subsequently removed from the transcriptomic data sets. The number of internal standard sequences recovered was used to estimate the absolute number of mRNA transcripts in the sample, and sample sequencing depth was defined as the actual number of mRNA reads sequenced in metatranscriptome divided by the absolute number of mRNA transcripts in the sample (17).

Reference genome assemblies for the four isolates that were used as inocula in the mesocosms were downloaded from NCBI (Table 1). Prodigal v2.6.1 (39) was used to predict genes from the assemblies, which were then annotated against the Swiss-Prot database (downloaded March 2019 [40]) using blastp (options: evalue 1E-6 and max_target_seqs 10 [41]). Matches to the reference Swiss-Prot sequences were filtered for best matching, using $40 \%$ identity and $40 \%$ query cover alignment length as threshold values. All genes that had no match to the Swiss-Prot database were annotated against the TrEMBL database (downloaded May 2018 [40]) using the same match filtering cutoff. Non-rRNA metatranscriptomic reads (i.e., after removal of internal standard sequences) were mapped against predicted genes using MegaBLAST (41) for the corresponding isolate that was used as an inoculum in that sample, and matches with $<97 \%$ identity and $<50$-bp alignment length were removed from further analysis. Read count tables against predicted genes were generated using custom scripts and were used as the input for DESeq2 v1.16.1 (42), and the sample sequencing depth as determined from the internal standard was used for the estimate size factor step. Differentially expressed genes between enteric and environmental isolates were determined using the likelihood ratio test and false-discovery rate $\left(P_{\text {adj }}<0.05\right)$ as implemented in DESeq2.

rRNA:rDNA ratio of $E$. faecalis in pure culture under standard laboratory conditions: batch culture growth conditions and sampling. E. faecalis strain MTUP9 (Table 1) was streaked for single colonies onto a TSA plate and grown overnight at $37^{\circ} \mathrm{C}$. A single colony was then inoculated into $4 \mathrm{ml}$ of TSB and incubated at $37^{\circ} \mathrm{C}$ with shaking at $150 \mathrm{rpm}$ for $14 \mathrm{~h}$. The overnight liquid culture (100 $\left.\mu \mathrm{l}\right)$ was inoculated into $60 \mathrm{ml}$ of fresh TSB in triplicate cultures to start the growth curve experiment (optical density at $600 \mathrm{~nm}\left[\mathrm{OD}_{600}\right]<0.1$ at time zero) and incubated at $37^{\circ} \mathrm{C}$ with shaking at $150 \mathrm{rpm}$. Each triplicate culture was sampled at 14 time points over $73 \mathrm{~h}$ to capture the different growth phases. At each sampling point, $1 \mathrm{ml}$ of each triplicate culture was collected for $\mathrm{OD}_{600}$ reading, $0.1 \mathrm{ml}$ was serially diluted 10 -fold in PBS for plate counts on TSA, and 0.5 to $1 \mathrm{ml}$ of the culture was collected for nucleic acid extraction by centrifugation at 10,000 rpm for $5 \mathrm{~min}$ and decanting of the supernatant. Cell pellets were resuspended in $600 \mu \mathrm{l}$ of buffer RLT (Qiagen) with $1 \%$ beta-mercaptoethanol and stored at $-80^{\circ} \mathrm{C}$ until ready for extraction. The resuspended cell pellets were defrosted on ice and transferred to 2-ml screwcap tubes prefilled with $100 \mathrm{mg}$ of acid-washed $0.1-\mathrm{mm}$ beads. Total nucleic acids were extracted and used for rRNA:rDNA analysis following the same protocol for filters as described above.

Data availability. Metatranscriptomic short reads have been deposited to the NCBI SRA database under BioProject no. PRJNA720051.

\section{SUPPLEMENTAL MATERIAL}

Supplemental material is available online only.

SUPPLEMENTAL FILE 1, PDF file, 0.2 MB.

\section{ACKNOWLEDGMENTS}

This work was supported by the U.S. National Science Foundation, award numbers 1511825 (to J.B. and K.T.K.) and 1831582 (K.T.K.), and the U.S. National Science Foundation Graduate Research Fellowship under grant number DGE-1650044 (to B.S.).

The funding agencies had no role in the study design, data collection and analysis, decision to publish, or preparation of the manuscript. The research presented was not performed or funded by EPA and was not subject to EPA's quality system requirements. The views expressed in this article are those of the author(s) and do not necessarily represent the views or the policies of the U.S. Environmental Protection Agency.

\section{REFERENCES}

1. Byappanahalli MN, Nevers MB, Korajkic A, Staley ZR, Harwood VJ. 2012. Enterococci in the environment. Microbiol Mol Biol Rev 76:685-706. https:// doi.org/10.1128/MMBR.00023-12.

2. Devane ML, Moriarty E, Weaver L, Cookson A, Gilpin B. 2020. Fecal indicator bacteria from environmental sources; strategies for identification to improve water quality monitoring. Water Res 185:116204. https://doi.org/10.1016/j .watres.2020.116204.

3. Mote BL, Turner JW, Lipp EK. 2012. Persistence and growth of the fecal indicator bacteria enterococci in detritus and natural estuarine plankton communities. Appl Environ Microbiol 78:2569-2577. https://doi.org/10.1128/AEM.06902-11. 
4. Weigand MR, Ashbolt NJ, Konstantinidis KT, Santo Domingo JW. 2014. Genome sequencing reveals the environmental origin of enterococci and potential biomarkers for water quality monitoring. Environ Sci Technol 48:3707-3714. https://doi.org/10.1021/es4054835.

5. Cesare AD, Pasquaroli S, Vignaroli C, Paroncini P, Luna GM, Manso E, Biavasco F. 2014. The marine environment as a reservoir of enterococci carrying resistance and virulence genes strongly associated with clinical strains. Environ Microbiol Rep 6:184-190. https://doi.org/10.1111/1758 -2229.12125 .

6. He Q, Hou Q, Wang Y, Li J, Li W, Kwok L-Y, Sun Z, Zhang H, Zhong Z. 2018. Comparative genomic analysis of Enterococcus faecalis: insights into their environmental adaptations. BMC Genomics 19:527. https://doi.org/10.1186/ s12864-018-4887-3.

7. Kemp PF, Lee S, LaRoche J. 1993. Estimating the growth rate of slowly growing marine bacteria from RNA content. Appl Environ Microbiol 59: 2594-2601. https://doi.org/10.1128/aem.59.8.2594-2601.1993.

8. Kerkhof L, Ward BB. 1993. Comparison of nucleic acid hybridization and fluorometry for measurement of the relationship between RNA/DNA ratio and growth rate in a marine bacterium. Appl Environ Microbiol 59:1303-1309. https://doi.org/10.1128/aem.59.5.1303-1309.1993.

9. Muttray AF, Mohn WW. 1999. Quantitation of the population size and metabolic activity of a resin acid degrading bacterium in activated sludge using slot-blot hybridization to measure the rRNA:rDNA ratio. Microb Ecol 38:348-357. https://doi.org/10.1007/s002489901005.

10. Kamke J, Taylor MW, Schmitt S. 2010. Activity profiles for marine spongeassociated bacteria obtained by $16 \mathrm{~S}$ rRNA vs $16 \mathrm{~S}$ rRNA gene comparisons. ISME J 4:498-508. https://doi.org/10.1038/ismej.2009.143.

11. Blazewicz SJ, Barnard RL, Daly RA, Firestone MK. 2013. Evaluating rRNA as an indicator of microbial activity in environmental communities: limitations and uses. ISME J 7:2061-2068. https://doi.org/10.1038/ismej.2013.102.

12. Flärdh K, Cohen PS, Kjelleberg S. 1992. Ribosomes exist in large excess over the apparent demand for protein synthesis during carbon starvation in marine Vibrio sp. strain CCUG 15956. J Bacteriol 174:6780-6788. https://doi.org/ 10.1128/jb.174.21.6780-6788.1992.

13. Worden AZ, Binder BJ. 2003. Growth regulation of rRNA content in Prochlorococcus and Synechococcus (marine cyanobacteria) measured by whole-cell hybridization of rRNA-targeted peptide nucleic acids. J Phycol 39:527-534. https://doi.org/10.1046/j.1529-8817.2003.01248.x.

14. Sukenik A, Kaplan-Levy RN, Welch JM, Post AF. 2012. Massive multiplication of genome and ribosomes in dormant cells (akinetes) of Aphanizomenon ovalisporum (Cyanobacteria). ISME J 6:670-679. https://doi.org/ 10.1038/ismej.2011.128.

15. Kapoor V, Pitkänen T, Ryu H, Elk M, Wendell D, Santo Domingo JW. 2015. Distribution of human-specific Bacteroidales and fecal indicator bacteria in an urban watershed impacted by sewage pollution, determined using RNA- and DNA-based quantitative PCR assays. Appl Environ Microbiol 81: 91-99. https://doi.org/10.1128/AEM.02446-14.

16. Goris J, Konstantinidis KT, Klappenbach JA, Coenye T, Vandamme P, Tiedje JM. 2007. DNA-DNA hybridization values and their relationship to whole-genome sequence similarities. Int J Syst Evol Microbiol 57:81-91. https://doi.org/10.1099/ijs.0.64483-0.

17. Satinsky BM, Gifford SM, Crump BC, Moran MA. 2013. Use of internal standards for quantitative metatranscriptome and metagenome analysis. Methods Enzymol 531:237-250. https://doi.org/10.1016/B978-0-12 -407863-5.00012-5.

18. Abrantes MC, Lopes M de F, Kok J. 2011. Impact of manganese, copper and zinc ions on the transcriptome of the nosocomial pathogen Enterococcus faecalis V583. PLoS One 6:e26519. https://doi.org/10.1371/journal .pone.0026519.

19. Lankiewicz TS, Cottrell MT, Kirchman DL. 2016. Growth rates and rRNA content of four marine bacteria in pure cultures and in the Delaware estuary. ISME J 10:823-832. https://doi.org/10.1038/ismej.2015.156.

20. Heim S, Lleo MDM, Bonato B, Guzman CA, Canepari P. 2002. The viable but nonculturable state and starvation are different stress responses of Enterococcus faecalis, as determined by proteome analysis. J Bacteriol 184:6739-6745. https://doi.org/10.1128/JB.184.23.6739-6745.2002.

21. US EPA. 2012. Recreational water quality criteria. EPA820-F-12-058. US EPA, Washington, DC.

22. Boehm AB, Graham KE, Jennings WC. 2018. Can we swim yet? Systematic review, meta-analysis, and risk assessment of aging sewage in surface waters. Environ Sci Technol 52:9634-9645. https://doi.org/10.1021/acs .est.8b01948.

23. Lleò MM, Pierobon S, Tafi MC, Signoretto C, Canepari P. 2000. mRNA detection by reverse transcription-PCR for monitoring viability over time in an Enterococcus faecalis viable but nonculturable population maintained in a laboratory microcosm. Appl Environ Microbiol 66:4564-4567. https://doi.org/10.1128/AEM.66.10.4564-4567.2000.

24. Signoretto C, Lleò MM, Tafi MC, Canepari P. 2000. Cell wall chemical composition of Enterococcus faecalis in the viable but nonculturable state. Appl Environ Microbiol 66:1953-1959. https://doi.org/10.1128/AEM.66.5 .1953-1959.2000.

25. Desmarais TR, Solo-Gabriele HM, Palmer CJ. 2002. Influence of soil on fecal indicator organisms in a tidally influenced subtropical environment. Appl Environ Microbiol 68:1165-1172. https://doi.org/10.1128/AEM.68.3 .1165-1172.2002

26. Roller BRK, Stoddard SF, Schmidt TM. 2016. Exploiting rRNA operon copy number to investigate bacterial reproductive strategies. 11. Nat Microbiol 1:16160-16167. https://doi.org/10.1038/nmicrobiol.2016.160.

27. Johnston ER, Kim M, Hatt JK, Phillips JR, Yao Q, Song Y, Hazen TC, Mayes MA Konstantinidis KT. 2019. Phosphate addition increases tropical forest soil respiration primarily by deconstraining microbial population growth. Soil Biol Biochem 130:43-54. https://doi.org/10.1016/j.soilbio.2018.11.026.

28. Neidhardt FC, Magasanik B. 1960. Studies on the role of ribonucleic acid in the growth of bacteria. Biochim Biophys Acta 42:99-116. https://doi.org/10 .1016/0006-3002(60)90757-5.

29. Wagner R. 1994. The regulation of ribosomal RNA synthesis and bacterial cell growth. Arch Microbiol 161:100-109. https://doi.org/10.1007/BF00276469.

30. Binder BJ, Liu YC. 1998. Growth rate regulation of rRNA content of a marine Synechococcus (cyanobacterium) strain. Appl Environ Microbiol 64: 3346-3351. https://doi.org/10.1128/AEM.64.9.3346-3351.1998.

31. Sobek JM, Charba JF, Foust WN. 1966. Endogenous metabolism of Azotobacter agilis. J Bacteriol 92:687-695. https://doi.org/10.1128/jb.92.3 .687-695.1966.

32. Oda Y, Slagman S-J, Meijer WG, Forney LJ, Gottschal JC. 2000. Influence of growth rate and starvation on fluorescent in situ hybridization of Rhodopseudomonas palustris. FEMS Microbiol Ecol 32:205-213. https://doi.org/ 10.1111/j.1574-6941.2000.tb00713.x.

33. Whitman RL, Shively DA, Pawlik H, Nevers MB, Byappanahalli MN. 2003. Occurrence of Escherichia coli and enterococci in Cladophora (Chlorophyta) in nearshore water and beach sand of Lake Michigan. Appl Environ Microbiol 69:4714-4719. https://doi.org/10.1128/AEM.69.8.4714-4719.2003.

34. Pitkänen $T$, Ryu $H$, Elk M, Hokajärvi A-M, Siponen S, Vepsäläinen A, Räsänen P, Santo Domingo JW. 2013. Detection of fecal bacteria and source tracking identifiers in environmental waters using rRNA-based RT-qPCR and rDNAbased qPCR assays. Environ Sci Technol 47:13611-13620. https://doi.org/10 $.1021 /$ es403489b.

35. Tsementzi D, Poretsky R, Rodriguez RL, Luo C, Konstantinidis KT. 2014. Evaluation of metatranscriptomic protocols and application to the study of freshwater microbial communities. Environ Microbiol Rep 6:640-655. https://doi.org/10.1111/1758-2229.12180.

36. Santo Domingo JW, Siefring SC, Haugland RA. 2003. Real-time PCR method to detect Enterococcus faecalis in water. Biotechnol Lett 25:261-265. https:// doi.org/10.1023/a:1022303118122.

37. Kim M, Hatt JK, Weigand MR, Krishnan R, Pavlostathis SG, Konstantinidis KT. 2018. Genomic and transcriptomic insights into how bacteria withstand high concentrations of benzalkonium chloride biocides. Appl Environ Microbiol 84:e00197-18. https://doi.org/10.1128/AEM.00197-18.

38. Kopylova E, Noé L, Touzet H. 2012. SortMeRNA: fast and accurate filtering of ribosomal RNAs in metatranscriptomic data. Bioinformatics 28:3211-3217. https://doi.org/10.1093/bioinformatics/bts611.

39. Hyatt D, Chen G-L, LoCascio PF, Land ML, Larimer FW, Hauser LJ. 2010. Prodigal: prokaryotic gene recognition and translation initiation site identification. BMC Bioinformatics 11:119. https://doi.org/10.1186/1471-2105-11-119.

40. UniProt Consortium. 2017. UniProt: the universal protein knowledgebase. Nucleic Acids Res 45:D158-D169. https://doi.org/10.1093/nar/gkw1099.

41. Camacho C, Coulouris G, Avagyan V, Ma N, Papadopoulos J, Bealer K, Madden TL. 2009. BLAST+: architecture and applications. BMC Bioinformatics 10:421. https://doi.org/10.1186/1471-2105-10-421.

42. Anders S, Huber W. 2010. Differential expression analysis for sequence count data. Genome Biol 11:R106. https://doi.org/10.1186/gb-2010-11-10-r106. 